International Journal on Cybernetics \& Informatics (IJCI) Vol. 5, No. 4, August 2016

\title{
Glaucoma Disease Diagnosis Using FeED FORWARD NEURAL NETWORK
}

\author{
Ch. P. Poojitha ${ }^{1}$, B. Nalini ${ }^{2}$ and Dr.N. Balaji ${ }^{3}$ \\ Department of Electronic and Communication Engineering, JNTUK - University College \\ of Engineering Vizianagaram, Vizianagaram, Andhra Pradesh
}

\begin{abstract}
Glaucoma is an eye disease which damages the optic nerve and or loss of the field of vision which leads to complete blindness caused by the pressure buildup by the fluid of the eye i.e. the intraocular pressure $(I O P)$. This optic disorder with a gradual loss of the field of vision leads to progressive and irreversible blindness, so it should be diagnosed and treated properly at an early stage. In this paper, thedaubechies(db3) or symlets (sym3)and reverse biorthogonal (rbio3.7) wavelet filters are employed for obtaining average and energy texture feature which are used to classify glaucoma disease with high accuracy. The Feed-Forward neural network classifies the glaucoma disease with an accuracy of 96.67\%. In this work, the computational complexity is minimized by reducing the number of filters while retaining the same accuracy.
\end{abstract}

\section{KEYWORDS}

Glaucoma, IOP, Wavelet transform, Texture features, Feed Forward neural network, Fundus images

\section{INTRODUCTION}

Glaucoma is found to be the second leading cause of irreversible visual loss and global blindness. Over the past decade, millions of cases of blindness have increased worldwide,gradually, of which $12.3 \%$ are of glaucoma. It has been estimated that nearly 80 million individuals around the world will suffer with glaucoma by the year 2020. Glaucoma is an ocular disease which causes progressive and irreversible changes in the visual field loss, optic nerve head, or both. The damage to the optic nerve is usually caused by raising Intraocular pressure (IOP) which is normally associated with increased fluid (aqueous humor) pressure in the eye (above $21 \mathrm{mmHg}$ ). The first sign of glaucoma is peripheral vision loss which often occurs gradually over a long period of time, so it is often called as "silent thief of sight", and symptoms only occur when the disease is quite advanced which requires early detection to sustain the vision without any further loss.

Different glaucoma detection tests include, Tonometry to determine IOP; Gonioscopy for finding the angle in the eye where the iris meets the cornea; Ophthalmoscopy/Funduscopy, it is a dilated eye examine for obtaining the shape and color of the optic nerve; Visual Field test (Perimetry) to check the missing areas of the field of vision; and Corneal Pachymetry for obtaining the thickness of nerve fiber layer. In this work, Ophthalmoscopy/Funduscopytest is considered asdiagnostic tool for the disease identification. Generally, the retinal fundus images are acquired by dilated eye test by exposing it to the light source and the images are captured using a fundus camera and microscope.

DOI: $10.5121 /$ ijci.2016.5417 
Many studies have been made for the development of automated clinical decision support systems (CDSSs) in ophthalmology, such as CASNET/glaucoma [1] and [2], which are designed for decision support system for disease identification in the eye.The features that can be extracted from the retinal fundus images which are used in these systems include structural and texture features. The frequently referred structural features are disk, rim and cup areas; disk and cup diameters and also the cup to disk ratio of the optic. The texture features measurement is based on the spatial variations of pixel intensity (gray-scale values) across the image[3]. In thesesystems, the proper orthogonal decomposition (POD) is one of the method that uses structural features for glaucoma identification [4]. The author in [5], used the texture and higher order spectra (HOS) features for glaucomatous image classification, in which HOS derives amplitude and phase information while texture provide measures such as smoothness, coarseness and regularity. The wavelet-Fourier analysis (WFA) [6] is used for obtaining the anatomic changes in the glaucoma where DWT used the symlets (sym4) wavelet filters for feature extraction by analyzing the abrupt changes in the signal while the fast Fourier transform (FFT) is applied to the detailed components, whose amplitudes are combined with the DWT normalized approximation coefficients to form the feature set.

The aim of this work is to automatically classify normal and glaucomatous eye images based on the wavelet based average and energy texture features obtained by applying the two wavelet family filters. Hence, the objective is to evaluate and select the potential features for improved specificity, sensitivity, precision and accuracy of the glaucomatous image classification. The proposed work uses the well-known wavelet family filters, the daubechies (db3), symlet (sym3) and reverse biorthogonal (rbio3. 7) combination filters are used to compute the wavelet average and energy features of the detailedcoefficients. The features which exhibits p-value $<0.0001$ are chosen and are subjected to z-score normalization to aid the convergence of feed forward neural network classifier. The paper is organized in the following way. Section 2 contains an explanation of the dataset. Section 3 contains a detailed description of the steps involved to detect glaucoma disease. Section 4 contains implementation and results along with the performance parameters employed in proposed methodology. In the end, the paper concludes with Section 5 with a conclusion.

\section{DATASET}

The fundus images of the retina used for this work are obtained from DRIVE and STARE databaseswhich are publicly available. All the images with resolution of $512 \times 512$ are taken in lossless JPEG format. The whole dataset comprises of 30 digital fundus images of which 15 are normal, which are taken from the DRIVE and other 15 are glaucomatous images taken from STARE database. The resulting retinal image shows the optic nerve, fovea, and the blood vessels. Figure 1 (a) and (b) representsnormal and glaucoma images, respectively.

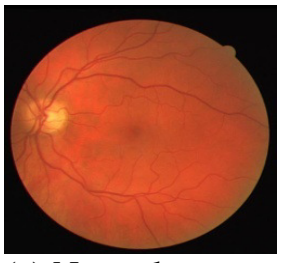

(a) Normal

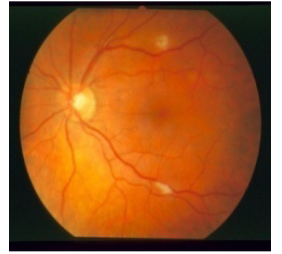

(b)Glaucoma

Figure 1.Retinal Fundus Images 
International Journal on Cybernetics \& Informatics (IJCI) Vol. 5, No. 4, August 2016

\section{Methodology}

The dataset containing all the images were initially preprocessed by subjecting them to standard histogram equalization. The processed images are then subjected to the following detailed procedure for feature extraction, feature selection and classification as shown in Figure 2.

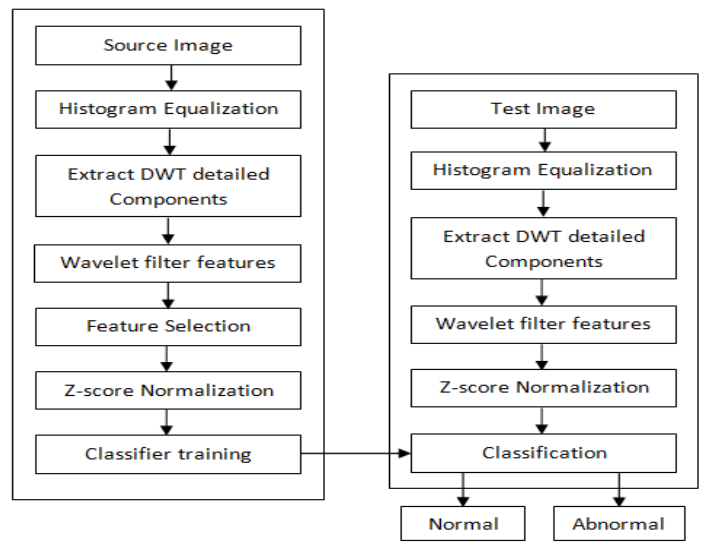

Figure 2. Flow of the methodology

\subsection{Preprocessing of images}

The dataset containing 30 images are preprocessed using standard Histogram equalization [7]. It is an image enhancement process and is required as the images are obtained from different subjects which have different orientations and intensity values so it is used to specify the input image pixel's intensity values, such that the output image contains intensity values which are uniformly distributed and also, the dynamic range of the image histogram is increased.

\subsection{Discrete Wavelet Transform based features}

Two dimensional Discrete wavelet transform (2D-DWT) is applied to the dataset to obtain the approximate and detail coefficients used for wavelet feature extraction. The one-level decomposition structure of an image of size $\mathrm{m} \times \mathrm{n}$ would result in four subimagesA1, Dh1, Dv1 and Dd1 each of size $\mathrm{m} / 2 \times \mathrm{n} / 2$ resolution and preserved scale.Here, the well-known wavelet family filters daubechies (db3)and reverse biorthogonal (rbio3.7) or symlets (sym3) and reverse biorthogonal (rbio3.7) combination filters are used for the feature extraction. Since the wavelet coefficient matrices obtained have a number of elements, to represent a feature in this work only a single value is required in orderto decrease the computation. Thus, these single-valued wavelet features are determined based on the averaging method using the DWT detailed coefficients are given by equations (1), (2) and (3) by averaging the intensity values. The equations (1) and (2) give the average features, while (3) gives the energy features. Here, $\mathrm{p} \times \mathrm{q}$ is the size of the subimages.

$$
\begin{aligned}
& \text { Average_Dh1 }=\frac{1}{p * q} \sum_{x=\{p\}} \sum_{y=\{q\}}|D h 1(x, y)| \\
& \text { Average_Dv1 }=\frac{1}{p * q} \sum_{x=\{p\}} \sum_{y=\{q\}}|D v 1(x, y)| \\
& \text { Energy_Dv1 }=\frac{1}{p^{2} * q^{2}} \sum_{x=\{p\}} \sum_{y=\{q\}}(D v 1(x, y))^{2}
\end{aligned}
$$


International Journal on Cybernetics \& Informatics (IJCI) Vol. 5, No. 4, August 2016

\subsection{P-values}

In this proposed work, 6 average and energy features each are extracted from the wavelet transformed fundus eye images for both the $\mathrm{db} 3 \&$ rbio3.7 and sym3 \& rbio3.7 combination filters. Among them, 6 features were chosen out of 12 features as it was proven to be clinically significant by using the p-value method which are same for-both filter combination are tabulated in Table 1. P-value method is a non-traditional statistical method used for hypothesis testing by comparing the two sample averages. In general, a p-value is the probability measure to determine the two mean differences which happened by chance.The statistical hypothesis is as follows:

$$
\begin{gathered}
H_{n}: \mu_{1}-\mu_{2}=0 \\
H_{a}: \mu_{1}-\mu_{2} \neq 0
\end{gathered}
$$

where, $H_{n}$ is the Null hypothesis which is the mean of the normal samples is the same as the mean of the Glaucoma samples and $\mathrm{H}_{\mathrm{a}}$ is the alternative hypothesis which is the mean of the normal samples is different than the mean of the Glaucoma samples. $\mu_{1}$ and $\mu_{2}$ are the means of normal and glaucoma samples.

Table 1. Selected Features

\begin{tabular}{|c|c|c|c|}
\hline Features & Normal & Glaucoma & P-Values \\
\hline db3_Dh_Average & $6.6415 \pm 0.8642$ & $4.3 .088 \pm 1.0183$ & $<0.0001$ \\
\hline db3_Dh_Energy & $0.0008 \pm 0.1758 \mathrm{E}-03$ & $0.3620 \mathrm{E}-03 \pm 0.1493 \mathrm{E}-03$ & $<0.0001$ \\
\hline db3_Dd_Energy & $0.0001 \pm 0.0383 \mathrm{E}-03$ & $0.0741 \mathrm{E}-03 \pm 0.0345 \mathrm{E}-03$ & $<0.0001$ \\
\hline rbio3.7_Dh_Average & $9.4660 \pm 1.2445$ & $6.0005 \pm 1.4257$ & $<0.0001$ \\
\hline rbio3.7_Dh_Energy & $0.0015 \pm 0.3525 \mathrm{E}-03$ & $0.7278 \mathrm{E}-03 \pm 0.3145 \mathrm{E}-03$ & $<0.0001$ \\
\hline rbio3.7_Dd_Energy & $0.0008 \pm 0.1958 \mathrm{E}-03$ & $0.4031 \mathrm{E}-03 \pm 0.1886 \mathrm{E}-03$ & $<0.0001$ \\
\hline
\end{tabular}

A two-sample t-test calculates the test statistics using equation (5) and (6) to estimate whether the mean of each of the average and energy features has significant clinical difference between the two groups, as

$$
\begin{aligned}
& t-s t a t=\frac{\overline{y_{1}}-\overline{y_{2}}}{\sqrt{s t d_{p}^{2}\left(\frac{1}{n_{1}}-\frac{1}{n_{2}}\right)}} \\
& s t d_{p}^{2}=\frac{\left(n_{1}-1\right) s_{1}^{2}-\left(n_{2}-1\right) s_{2}^{2}}{n_{1}+n_{2}-2}
\end{aligned}
$$

where, $\overline{y_{1}}$ and $\overline{y_{2}}$ are the means of the each class sample and $n_{1}$ and $n_{2}$ represents the sample sizes and $s_{\mathrm{p}}$ is the pool variance given as follows with $\mathrm{s}_{1}$ and $\mathrm{s}_{2}$ are the variances of the classes. Using the t-statistics, the p-value is calculated which is the area under the t-distribution curve past the $\mathrm{t}$ stats are compared with the level of significance $(\alpha=0.0001)$, the lower the $p$-value indicates that there is a clinical significantly greater difference between the two classes. Generally, $\mathrm{H}_{\mathrm{n}}$ is rejected, if the $\mathrm{p}$-value $<0.0001$ corresponding to $0.01 \%$ chance of null hypothesis being true. Hence, for further analysis the features which exhibit p-values less than 0.0001 are selected.

\subsection{Z-score Normalization of features}

The $\mathrm{z}$-score is a normalization process used for rescaling the features i.e. the average and energy feature into the same units. The 6 features selected are normalized using z-score normalization. In this process, a vector sample consisting of 6 features is transformed to unit variance and zero mean. The z-scores are computed using equation (7) with $v_{\text {old }}$ as the original value in the vector 
and $v_{\text {new }}$ is its obtained new z-score value, and $\mathrm{m}$ and Std are the mean and standard deviation of the original data range, respectively.

$$
v_{\text {new }}=\frac{v_{\text {old }}-m}{S t d}
$$

\subsection{Classification - Feed Forward Neural Network}

A network structure having only the advancing flow of information without any feedback is called a feed forward neural network(FFNN) [8]. One of the most widely preferred is multilayered feed forward network with back propagation shown in Figure 3, as a single artificial neuron cannot model well for classifications that are non-linearly separable.

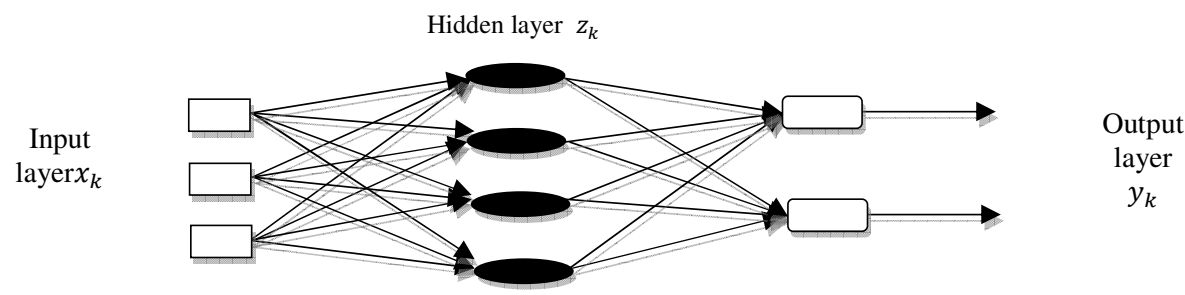

Figure 3.Multi layered feed forward network architecture

In this work, the features which are extracted are non-linear and are to be classified, so multi layered neural network is employed. From the figure it is clear that the network architecture has three layers that are input layer, hidden layer and output layer, where the hidden layer can be of any number depending upon the user usage, which can compute the input representation that will make the problem more linearly separable. This multi-layered network uses extended gradientdescent based delta-learning rule, commonly known as a back propagation rule as training algorithm. The training is performed by using a supervised learning technique, where back propagation is used for altering the weight using an activation function for learning a training set has been proved to be computationally efficient method and the total mean squared error of the weights are minimized using the gradient descent method.During this training process an iterative based flow of training data is followed for the weight vectors establishment, using which the network learns to identify particular classes by considering the characteristics of the input trained data and the backward links are present during training only. Finally, the activation function used is a linear sigmoid function, using which the output is as specified:

$$
y=f(x)=\frac{1}{1+e^{-x}}
$$

Using the sigmoid activation function binary classification can be performed as it will squash allthe output values between $0 \& 1$, such that the neuron will always be positive, bounded and is strictly increasing. The errors in the output nodes are propagated backward through the network after each training case and the system adjusts the weights of the net so that the total mean squareerror calculated using the delta rule is minimized. Once the system is optimized by training with the input pattern to obtain the trained features, the test images can be given which follow the same procedure, except the backward links, for detection of the image class i.e. glaucoma or normal retinal images can be achieved by categorizing them as either 0 or 1 . 


\section{IMPLEMENTATION AND RESULTS}

The proposed method for glaucoma detection was tested on a publicly available DRIVE and STARE database. The 6 wavelet features were extracted from each retinal image ( 1 x 6 matrix $)$ and thus for the whole dataset a $30 \times 6$ feature matrix was formed where each row corresponds to each image input and the 6 columns represents the selected features extracted from each image.This input vector of $30 \times 6$ feature matrix is then fed to the FFNN system with 120 hidden layers and a target matrix as the identity matrix of size 30 , since 30 image samples are used to classify the normal and glaucomatous image. Thus, after the network training phase, the test images are given whose features are extracted by undergoing the same procedures and then the trained set features are compared with the test features for classification of glaucoma.

The performance of the system is analyzed by evaluating the 4 parameters Sensitivity (TPR), Specificity (SPC), Positive Predictive Accuracy (PPV) or Precision and Accuracy (ACC) are calculated with respect to the four conditions which form the four entries of the confusion matrix are TP (true positive) for glaucoma is correctly identified, FP (false positive) for glaucoma is incorrectly identified, TN (true negative) for normal is correctly rejected, and FN (false negative) for normal is incorrectly rejected; where positive $(\mathrm{P})$ and negative $(\mathrm{N})$ corresponds to glaucoma and normal case respectively. The test results can be positive or negative corresponding to the identification or rejection of image pattern respectively. The equations for calculating these performance parameters are given below:

$$
\begin{aligned}
& \mathrm{TPR}=\mathrm{TP} /(\mathrm{TP}+\mathrm{FN}) \\
& \mathrm{SPC}=\mathrm{TN} /(\mathrm{FP}+\mathrm{TN}) \\
& \mathrm{PPV}=\mathrm{TP} /(\mathrm{TP}+\mathrm{FP}) \\
& \mathrm{ACC}=(\mathrm{TP}+\mathrm{TN}) /(\mathrm{P}+\mathrm{N})
\end{aligned}
$$

Sensitivity and specificity are both are the measures of the fraction of positives and negatives which wereidentified correctly by the classifier, respectively. Precision determines the fraction of samples that are positive in the group which has been declared as a positive class by the classifier. Accuracy is the whole system parameter which is used as a statistical measure to determine how well a binary classifier identifies or rejects a test sample. A system having $100 \%$ sensitivity and specificity is considered as a perfect predictor, evenso, theoretically some minimum error usually exists in any predictor.For the neural network systems, the obtained four conditions and performance parameters are tabulated in the Table 2.

Table 2. Performance parameters comparison

\begin{tabular}{|c|c|c|c|c|c|c|c|c|}
\hline $\begin{array}{c}\text { Filter } \\
\text { Combinations }\end{array}$ & TP & FP & TN & FN & $\begin{array}{c}\text { TPR } \\
(\%)\end{array}$ & $\begin{array}{c}\text { SPC } \\
(\%)\end{array}$ & $\begin{array}{c}\text { PPV } \\
(\%)\end{array}$ & $\begin{array}{c}\text { ACC } \\
(\%)\end{array}$ \\
\hline 5 - Filters & 14 & 1 & 15 & 0 & 100 & 93.75 & 93.33 & 96.67 \\
\hline 2 - Filters & 14 & 1 & 15 & 0 & 100 & 93.75 & 93.33 & 96.67 \\
\hline
\end{tabular}

In this research work an effort is made to reduce the number of wavelet filters to two, while retaining the accuracy of $96.67 \%$ for glaucoma image classification using five wavelet filters[10].In the proposed work, the computational complexity is reduced as the number of wavelet filters employed are less. In a two wavelet filter combination daubechies $(\mathrm{db} 3)$ and reverse biorthogonal (rbio3.7) or symlets (sym3) and reverse biorthogonal (rbio3.7) filters are usedand in the five filter combination includes daubechies (db3), symlet (sym3) and reverse 
biorthogonal (rbio3.3, rbio3.5 and rbio3.7) wavelet filters are employed [9][10].The two sets of two filter combinations using daubechies and symlets produces the same accuracy as both have same filter coefficient and filter lengths, so any one of the combination can be employed. These filters are biorthogonal filters with high pass and low pass filter lengths of $\mathrm{L}_{1}$ and $\mathrm{L}_{2}$, then the number of additions, A, and multiplications, M, required for performing the filter operations are computed [11] using the equation (13) and (14).These formulas compute the computational complexity of one dimensional signal, in order to calculate for a two-dimensional signal i.e. in case of an image, the formulas are multiplied by 2 to relate to the separable filtering of horizontal and vertical filtering. The complexity comparison for the filter combinations is shown in the Table 3 .

$$
\begin{aligned}
& \mathrm{M}=\mathrm{L}_{1}+\mathrm{L}_{2} \\
& \mathrm{~A}=\mathrm{L}_{1}+\mathrm{L}_{2}-2
\end{aligned}
$$

The work is implemented using MATLAB. Using a Graphical user interface (GUI), the image is selected for analysis is first preprocessed using histogram equalization and applied to daubechies and reverse biorthogonalwavelet filters. The option feature extraction provides the list of average and energy features which can be extracted from the images. The created GUI has two tables, one corresponding to all the texture features of the images in the database and the other displays the selected average and energy feature based on the p-value criteria as shown in the Figure 4.The classification option is selected to display the classification window to classify the images using the FFNN classifier where the tables shown in the Figure 5 and 6 are the four conditions to calculate the performance parameters. FFNN classifies the dataset images with an accuracy of $96.67 \%$, which is displayed on the GUI by selecting the accuracy option. The GUI also displays a message for glaucoma detection of the input retinal fundus images.

Table 3. Computational Complexity Comparison for 2D signal (image)

\begin{tabular}{|c|c|c|c|}
\hline \multirow{2}{*}{$\begin{array}{c}\text { Filter } \\
\text { Combinations }\end{array}$} & \multicolumn{3}{|c|}{ Computational Complexity } \\
\cline { 2 - 4 } & Multiplications & Additions & $\begin{array}{c}\text { No. of } \\
\text { Computations }\end{array}$ \\
\hline 5 - Filters & 72 & 52 & 124 \\
\hline 2 - Filters & 32 & 24 & 56 \\
\hline
\end{tabular}

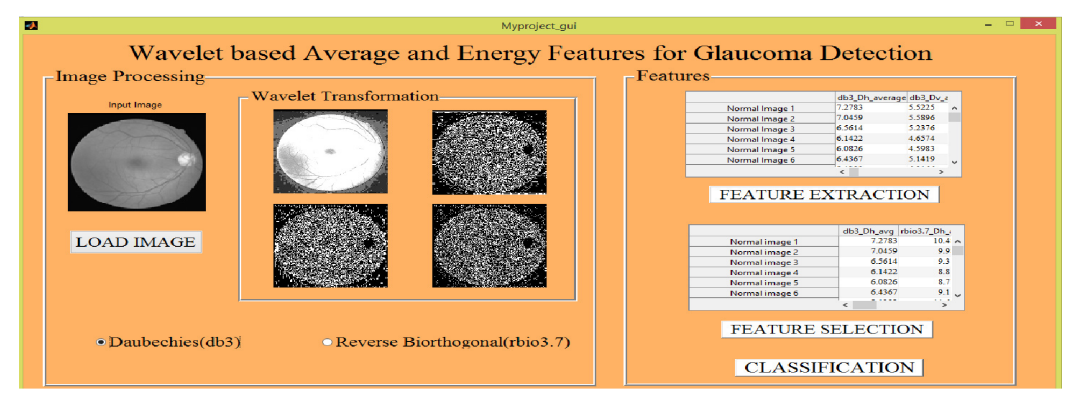

Figure 4. GUI for Glaucoma Detection 
International Journal on Cybernetics \& Informatics (IJCI) Vol. 5, No. 4, August 2016

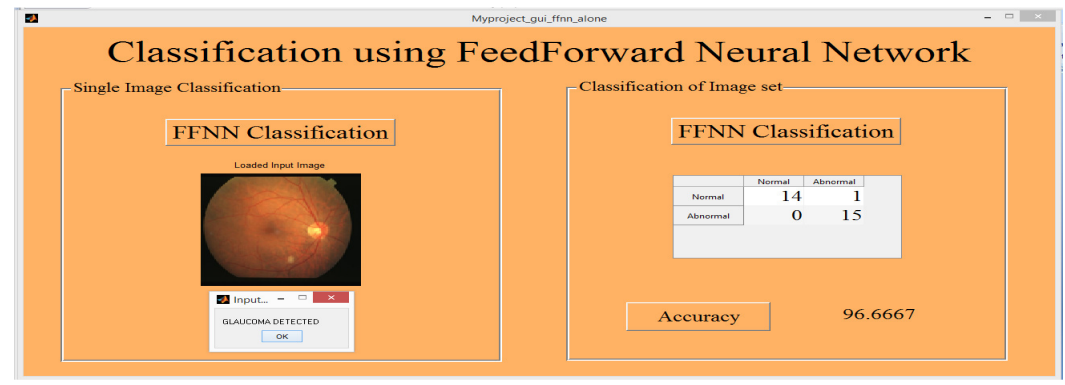

Figure 5. Glaucoma Image classification results

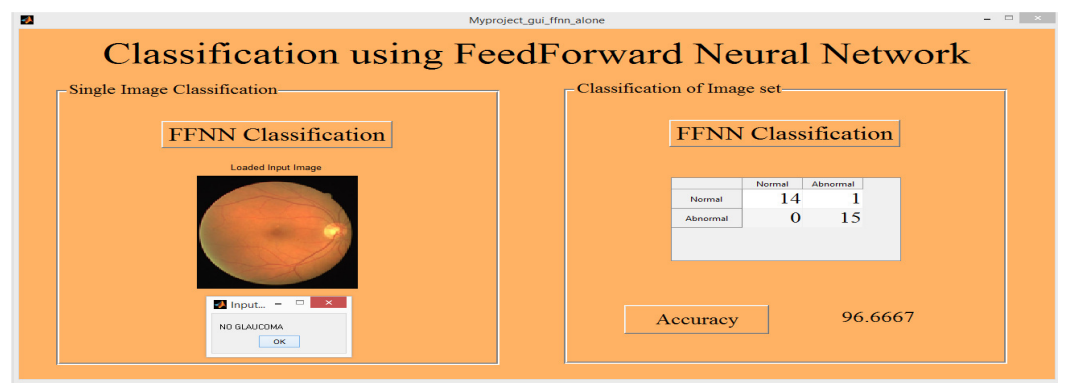

Figure 6. Normal Image classification results

\section{CONCLUSION}

This study illustrates that the selected features extracted from the two wavelet filters have been fed to a Feed Forward Neural Network. The texture features obtained from the detailed coefficients of the two wavelet filters are used to discriminatenormal and glaucomatous images by retaining the same accuracy of $96.67 \%$ as that of five filter combination and also reduces the computational complexity required for the classification

\section{REFERENCES}

[1] S. Weiss, C. A. Kulikowski and A. Safir, (1978) "Glaucoma consultation by computer," Comp. Biol. Med., vol. 8, pp. 24-40.

[2] S. Weiss et al., (1978) "A model-based method for computer-aided medical decision-making," Artif.Intell., vol. 11, pp. 145-172.

[3] R. O. Duncan et al., (Feb, 2007) "Retinotopic organization of primary visual cortex in glaucoma: A method for comparing cortical function with damage to the optic disk," Invest. Ophthalmol. Vis. Sci., vol. 48, pp. 733-744.

[4] M. Balasubramanian et al., (2010) "Clinical evaluation of the proper orthagonal decomposition framework for detecting glaucomatous changes in human subjects," Invest. Ophthalmol. Vis. Sci., vol. 51, pp. 264-271.

[5] U. R. Acharya, S. Dua, X. Du, V. S. Sree, and C. K. Chua, (May 2011)“Automated diagnosis of glaucoma using texture and higher order spectra features,” IEEE Trans. Inf. Technol. Biomed., vol. 15, no. 3, pp. 449-455.

[6] E. A. Essock, Y. Zheng, and P. Gunvant, (Aug. 2005) "Analysis of GDx-VCC polarimetry data by wavelet- Fourier analysis across glaucoma stages,” Invest. Ophthalmol. Vis. Sci., vol. 46, pp. 28382847.

[7] R. C. Gonzalez and R. E. Woods, (2001) Digital Image Processing. NJ: Prentice Hall. 
International Journal on Cybernetics \& Informatics (IJCI) Vol. 5, No. 4, August 2016

[8] Christopher M Bishop, "Neural Networks for pattern recognition", Dept of Computer Science and Applied Mathematics, Aston University, UK.

[9] SumeetDua, U. Rajendra Acharya, Pradeep Chowriappa\&S.VinithaSree, (2012) "Wavelet - Based Energy Features for Glaucomatous Image Classification", IEEE TRANSACTIONS on Information Technology In Biomedicine, Vol. 16, No: 1, pp 1089-7771 .

[10] Nisha Simon \&Hymavathy K. P., (2015) "Early stage Glaucoma Screening and Segmentation using FFNN", International Journal of Engigneering Research \& Technology, Vol. 4, No: 4, pp 783-788.

[11] Nikolay Polyak and William A Pearlman, "A New Flexible Biorthogonal Filter Design for Multiresolution Filterbanks with Application to Image Compression", To Appear in the IEEE Transactions on Signal Processing.

\section{AUTHORS}

Dr. N. Balaji obtained his B.Tech degree from Andhra University. He received his Master's and Ph.D. degree from Osmania University, Hyderabad. Currently he is working as HOD and a professor in the Department of ECE JNTUK University College of Engineering Vizianagaram. He has authored more than 30 research papers in National and International Conferences and Journals. He is a life member of ISTE and Member, Treasurer of VLSI Society of India Local Chapter, Hyderabad. His areas of research interest are VLSI, Signal Processing, Radar, and Embedded Systems.

B. Nalini received her B.Tech and Master's from JNTU Kakinada. Currently she is working as assistant professor in the Department of ECE JNTUK University College of Engineering Vizianagaram. Her areas of interests are VLSI and Biomedical Image Processing.

Ch. P. Poojitha received her B.Tech from Vignan's Institute of Information Technology, Andhra Pradesh. Currently she is pursuing her Master's in the specialization of Systems and Signals Processing in the Department of ECE JNTUK University College of Engineering Vizianagaram. 